\title{
Challenges and Limitations of Clinical Trials on Labor Induction: A Review of the Literature
}

\author{
Oscar A. Viteri, MD ${ }^{1}$ Baha M. Sibai, MD² \\ ${ }^{1}$ Avera Medical Group Maternal Fetal Medicine, Avera McKennan \\ Hospital and University Health Center, Sioux Falls, South Dakota \\ 2 Department of Obstetrics, Gynecology and Reproductive Sciences, \\ UTHealth McGovern Medical School, Houston, Texas \\ Am J Perinatol Rep 2018;8:e365-e378.
}

\begin{abstract}
Address for correspondence Oscar A. Viteri, MD, Avera McKennan Hospital and University Health Center, 1417 S Cliff Ave. Suite 100, Sioux Falls, South Dakota 57105 (e-mail: oaviteri.md@gmail.com).
\end{abstract}

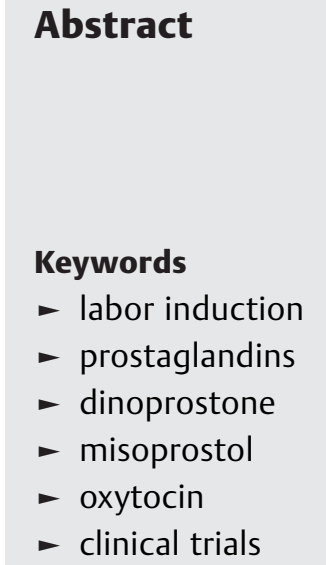

Induction of labor is a common obstetric procedure performed in nearly a quarter of all deliveries in the United States. Pharmacological (prostaglandins, oxytocin) and/or mechanical methods (balloon catheters) are commonly used for labor induction; however, there is ongoing debate as to which method is the safest and most effective. This narrative review discusses key limitations of published trials on labor induction, including the lack of well-designed randomized controlled trials directly comparing specific methods of induction, heterogeneous trial populations, and wide variation in the protocols used and outcomes reported. Furthermore, the majority of published trials were underpowered to detect significant differences in the most clinically relevant efficacy and safety outcomes (e.g., cesarean delivery, neonatal mortality). By identifying the limitations of labor induction trials, we hope to highlight the importance of quality published data to better inform guidelines and drive evidence-based treatment decisions.
Induction of labor is a common obstetric procedure that is used in nearly a quarter (24.5\%) of all deliveries in the United States (U.S.). ${ }^{1}$ Induction of labor is indicated when the risks to maternal or fetal health outweigh the benefits of continuing the pregnancy. ${ }^{2}$ One of the most common reasons for labor induction in the U.S. is postterm pregnancy (i.e., $>41$ weeks of gestation). ${ }^{3}$ Other indications for labor induction include chorioamnionitis, gestational hypertension, preeclampsia, premature rupture of membranes (PROM), maternal conditions (e.g., diabetes mellitus, chronic hypertension), and fetal compromise (e.g., severe fetal growth restriction, oligohydramnios). ${ }^{2}$ Women may also choose to undergo elective labor induction (i.e., inductions performed without maternal or fetal medical indication) to shorten the duration of pregnancy or to schedule the day of delivery. ${ }^{4}$ In the U.S., elective inductions account for approximately $10 \%$ of all labor inductions. ${ }^{5-7}$

The process of labor induction often begins with cervical ripening which involves softening and thinning the cervix in preparation for labor and delivery. Different methods are available for cervical ripening and labor induction, including pharmacological and/or mechanical methods. ${ }^{2,8,9}$ Oxytocin, a hormone that stimulates uterine contractions, is considered less effective for cervical ripening and is typically used alone for labor induction when the cervix is favorable (Bishop's score $\geq 6$ ) or after cervical ripening with prostaglandins or mechanical methods., ${ }^{2,10}$ The use of oxytocin alone for cervical ripening has been associated with higher rates of unsuccessful vaginal deliveries within 24 hours and increased rates of cesarean delivery compared with the use of prostaglandins. ${ }^{11}$ Dinoprostone (prostaglandin E2) is the only prostaglandin approved by the U.S. Food and Drug Administration (FDA) for cervical ripening and induction of labor. ${ }^{12,13}$ Misoprostol (prostaglandin E1 analog) is widely used for labor induction, although it is not approved by the FDA. ${ }^{14}$ Mechanical methods of labor induction (e.g., Foley's balloon catheters, double-balloon catheters, hygroscopic and osmotic dilators) are effective alone but are also commonly combined with pharmacological methods. Oxytocin, both prostaglandin preparations, and balloon catheters are received

June 15, 2018

accepted after revision

October 12, 2018
DOI https://doi.org/

10.1055/s-0038-1676577. ISSN 2157-6998.
Copyright $\odot 2018$ by Thieme Medical Publishers, Inc., 333 Seventh Avenue, New York, NY 10001, USA. Tel: +1(212) 584-4662.
License terms

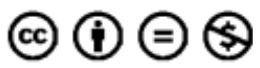


recommended by the American College of Obstetricians and Gynecologists (ACOG) and World Health Organization (WHO) for labor induction. ${ }^{2,4}$

There are several potential maternal and neonatal complications with labor induction, although mechanical methods, such as the Foley catheter, are associated with the least number of adverse effects. ${ }^{10}$ Previous trials have suggested that women undergoing induction of labor with pharmacological and/or mechanical methods may be at a higher risk of cesarean section. However, recent analyses dispute the association between labor induction and increased risk of a cesarean delivery. ${ }^{15-17}$ For example, preliminary data from a recent large, randomized, controlled trial (RCT) in healthy nulliparous women comparing elective induction of labor at 39 weeks versus expectant management (A Randomized Trial of Induction Versus Expectant Management [ARRIVE]; $n=6,106$ ) showed a reduction in cesarean delivery rates with labor induction versus expectant management (18.6 vs. $22.2 \%$; relative risk $[R R]=0.84 ; 95 \%$ confidence interval $[\mathrm{CI}]$ : $0.76-0.93) .^{18}$ of note, rates of the composite primary outcome of adverse perinatal events were similar in women undergoing labor induction versus those in the expectant management group (4.3 vs. $5.4 \%$; $R R=0.80$; 95\% CI: 0.64-1.00). ${ }^{18}$

While ACOG recommends several methods for labor induction, ${ }^{2}$ the safest and most effective method has not yet been clearly established. Although effective, pharmacological methods of labor induction have been associated with potential risks, such as uterine tachysystole with or without fetal heart rate changes, uterine rupture, fetal distress, and maternal and perinatal morbidity and mortality. $^{2,19-22}$ The incidence of these adverse events is dependent on the agent used for induction, dose, and other factors. The appropriate method of induction, whether pharmacological and/or mechanical, may vary depending on maternal and neonatal factors, patient demographics, and other variables that can complicate clinical trial design and present a barrier to evidence-based recommendations. Clinical trials in labor induction are further limited by the lack of uniform protocols, inconsistent and incomplete reporting of outcomes, and lack of statistical power for clinically relevant efficacy and safety outcomes, such as cesarean delivery rates or severe neonatal morbidity and mortality. These limitations make it difficult to interpret trial data and draw meaningful conclusions about which method of labor induction is appropriate based on indication and other patient-related characteristics.

This narrative review discusses the limitations of published clinical trials on labor induction and highlights fundamental issues in trial design and methodology that can impact the interpretation of results. We provide recommendations for future trials on labor induction, including important components of a rigorous, well-designed, and properly conducted clinical trial. We hope that these recommendations will yield quality data to better inform guidelines and drive evidence-based practice to help physicians make better treatment decisions.

\section{Methods}

A comprehensive literature search was conducted for this narrative review using PubMed and the search terms "labor AND induction" or "labor AND delivery AND vaginal," including both the U.S. and British spelling of "labor" and "labour." The search was limited to English language RCTs, prospective trials, and observational studies published between January 2007 and October 2017.

A total of 548 titles and abstracts were manually screened for inclusion. Clinical trials of pharmacological (i.e., prostaglandins, oxytocin) and mechanical methods of labor induction were included in the review. Exclusion criteria included: retrospective studies; descriptions of trial protocols (i.e., no efficacy or safety data reported); secondary analyses; intra dose-comparison trials; trials of alternative methods of labor induction (i.e., acupuncture, nipple stimulation, nitric oxide donors [isosorbide mononitrate, isonicotinic acid hydrazide], dexamethasone, propranolol); trials with outpatient agent administration; trials in women with PROM, preterm PROM (pPROM), or trial of labor after cesarean (TOLAC); trials of induction for abortion or fetal death; and any other off-topic trials that did not compare methods of labor induction (e.g., studies to identify predictors of successful labor induction, methods for pain management during induction, and pharmacokinetic studies).

\section{Summary of Literature Search Results}

A total of 103 clinical trials met the inclusion and exclusion criteria; 63 were RCTs, 21 were nonrandomized prospective trials, and 19 were observational studies. Of the 63 RCTs, ${ }^{23-85}$ most were single-center trials conducted outside the U.S. that enrolled a limited number of participants. Just 12 RCTs were conducted in the U.S. (- Table 1). The inclusion criteria for most trials were nulliparous or multiparous women with single, cephalic pregnancies and obstetric or medical indications for induction of labor. The most common primary outcomes were time from the start of induction to delivery and vaginal delivery within 24 hours. The reporting of safety outcomes varied, yet all trials included at least some maternal and/or neonatal safety data.

The published clinical trials on labor induction, including trials conducted within and outside the U.S., have many limitations that are both inherent to the trial designs and a result of the therapeutic area and patient population. These limitations are discussed in detail in the following section and are summarized in - Table 2 .

\section{Limitations of Trials on Labor Induction}

\section{Trial Design, Sample Size, Randomization, and Blinding}

Various clinical trial designs were identified in the literature search, including observational trials, nonrandomized prospective trials, and RCTs. Of the 103 trials identified, just 63 were RCTs. $^{23-85}$ The majority of RCTs were single-center trials conducted in academic hospitals, which can limit the 
Table 1 Summary of published labor induction RCTs in the United States

\begin{tabular}{|c|c|c|c|c|c|}
\hline Clinical trial & Drug/device & Trial design & Trial population & Outcomes & Main results \\
\hline $\begin{array}{l}\text { Schoen et al (2017), } \\
\text { intracervical Foley } \\
\text { catheter with and with- } \\
\text { out oxytocin for labor } \\
\text { induction: a randomized } \\
\text { controlled trial }{ }^{25}\end{array}$ & $\begin{array}{l}\text { Foley's catheter with } \\
\text { concurrent oxytocin } \\
\text { infusion vs. Foley's } \\
\text { catheter followed by } \\
\text { oxytocin infusion } \\
\text { Dosing: } \\
\text { - } 16 \mathrm{~F} \text { or } 20 \mathrm{~F} \text { Foley's } \\
\text { catheter inflated to } \\
60 \mathrm{~mL} \\
\text { - Oxytocin infusion } \\
\text { started at } 2 \mathrm{mU} / \mathrm{min} \\
\text { and increased by } \\
2 \mathrm{mU} / \mathrm{min} \text { after } \\
30 \mathrm{~min} \text {, max } \\
40 \mathrm{mU} / \mathrm{min}\end{array}$ & Multicenter parallel RCT & $\begin{array}{l}n=323 \\
\text { - Nulliparous and } \\
\text { multiparous women } \\
\text { with a singleton } \\
\text { pregnancy at } \\
\geq 24 \text { wk } \\
\text { - Bishop's score }<6 \\
\text { - Women with pPROM } \\
\text { were not excluded }\end{array}$ & $\begin{array}{l}\text { Primary: } \\
\text { - Delivery } \leq 24 \mathrm{~h} \\
\text { - Secondary: } \\
\text { - Time to Foley's expul- } \\
\text { sion } \\
\text { - Change in Bishop's } \\
\text { score } \\
\text { - Need for additional } \\
\text { ripening } \\
\text { - Analgesia during } \\
\text { Foley's catheter use } \\
\text { - Time to second stage } \\
\text { - Delivery } \leq 12 \text { h } \\
\text { - Total time to delivery } \\
\text { - Duration of oxytocin } \\
\text { use } \\
\text { - Mode of delivery } \\
\text { - Tachysystole } \\
\text { - Chorioamnionitis } \\
\text { - Postpartum hemor- } \\
\text { rhage } \\
\text { - Severe maternal mor- } \\
\text { bidity (uterine rupture, } \\
\text { ICU admission, or } \\
\text { maternal death) } \\
\text { - Neonatal outcomes } \\
\text { (weight, 5-min Apgar's } \\
\text { score }<7, \text { NICU } \\
\text { admission, NICU length } \\
\text { of stay) }\end{array}$ & $\begin{array}{l}\text { - More nulliparous (64 } \\
\text { vs. } 43 \% ; p=0.003 \text { ) } \\
\text { women who received } \\
\text { Foley's catheter with } \\
\text { concurrent oxytocin } \\
\text { delivered } \leq 24 \text { h vs. } \\
\text { Foley's catheter fol- } \\
\text { lowed by oxytocin } \\
\text { - Median time to deliv- } \\
\text { ery was shorter in both } \\
\text { nulliparous ( } 20.9 \text { vs. } \\
26.1 \text { h; } p<0.001 \text { ) and } \\
\text { multiparous ( } 14.9 \text { vs. } \\
18.6 \text { h; } p=0.01 \text { ) } \\
\text { women who received } \\
\text { Foley's catheter with } \\
\text { concurrent oxytocin } \\
\text { - No significant differ- } \\
\text { ences in cesarean sec- } \\
\text { tion rates, postpartum } \\
\text { hemorrhage, chor- } \\
\text { ioamnionitis, or NICU } \\
\text { admission }\end{array}$ \\
\hline $\begin{array}{l}\text { Connolly et al, a rando- } \\
\text { mized trial of Foley bal- } \\
\text { loon induction of labor } \\
\text { trial in nulliparas } \\
(\text { FIAT-N) }\end{array}$ & $\begin{array}{l}\text { Foley's catheter with } \\
\text { concurrent oxytocin } \\
\text { infusion vs. Foley's } \\
\text { catheter followed by } \\
\text { oxytocin infusion } \\
\text { Dosing: } \\
\text { - } 16 \mathrm{~F} \text { Foley's catheter } \\
\text { inflated to } 60 \mathrm{~mL} \\
\text { - Oxytocin infusion } \\
\text { started at } 2 \mathrm{mU} / \mathrm{min} \\
\text { and doubled every } \\
30 \text { min to a max dose } \\
\text { of } 16 \mathrm{mU} / \mathrm{min} \text {; then } \\
\text { increased by } 2 \mathrm{mU} / \\
\text { min every } 30 \mathrm{~min} \text {, } \\
\text { max dose } 30 \mathrm{mU} / \mathrm{min}\end{array}$ & Single-center RCT & $\begin{array}{l}n=166 \\
\text { - Nulliparous women } \\
\text { with a singleton } \\
\text { pregnancy at } \\
\geq 24 \text { wk gestation } \\
\text { - Cervical dilation } \\
<3 \mathrm{~cm}\end{array}$ & $\begin{array}{l}\text { Primary: } \\
\text { - Time to delivery } \\
\text { Secondary: } \\
\text { - Cesarean delivery rate } \\
\text { - Chorioamnionitis } \\
\text { - Estimated blood loss } \\
\text { - Postpartum hemor- } \\
\text { rhage } \\
\text { - Composite neonatal } \\
\text { outcome ( } \geq 1 \text { of } \\
5 \text {-min Apgar's score } \\
<5, \text { umbilical artery } \\
\text { pH }<7.1 \text {, NICU admis- } \\
\text { sion, NEC, or neonatal } \\
\text { death) }\end{array}$ & $\begin{array}{l}\text { - Women who received } \\
\text { Foley's catheter with } \\
\text { concurrent oxytocin } \\
\text { had a shorter mean } \\
\text { time to delivery vs. } \\
\text { Foley's catheter fol- } \\
\text { lowed by oxytocin } \\
(15.9 \text { vs. } 18.9 \text { h; } \\
p=0.004) \\
\text { - No significant differ- } \\
\text { ences in cesarean } \\
\text { delivery rates, chor- } \\
\text { ioamnionitis, esti- } \\
\text { mated blood loss, } \\
\text { postpartum hemor- } \\
\text { rhage, or composite } \\
\text { neonatal outcome }\end{array}$ \\
\hline $\begin{array}{l}\text { Levine et al, mechanical } \\
\text { and pharmacologic } \\
\text { methods of labor induc- } \\
\text { tion: a randomized con- } \\
\text { trolled trial }\end{array}$ & $\begin{array}{l}\text { Foley's catheter plus } \\
\text { vaginal misoprostol } \\
\text { tablet vs. Foley's cathe- } \\
\text { ter plus oxytocin infu- } \\
\text { sion vs. Foley's catheter } \\
\text { alone vs. vaginal miso- } \\
\text { prostol tablet alone } \\
\text { Dosing: } \\
\text { - } 18 \mathrm{~F} \text { Foley's catheter } \\
\text { inflated to } 60 \mathrm{~mL} \\
\text { - } 25 \mu \mathrm{g} \text { vaginal miso- } \\
\text { prostol tablet given } \\
\text { every } 3 \text { h, repeated } \\
\text { up to five times for a } \\
\text { max of } 24 \mathrm{~h} \\
\text { - Oxytocin infusion } \\
\text { started at } 2 \text { mU/min } \\
\text { and increased by } 2 \\
\text { mu/min every } \\
15 \mathrm{~min}, \text { max dose } \\
40 \mathrm{mU} / \text { min }\end{array}$ & Single-center RCT & $\begin{array}{l}n=491 \\
\text { - Nulliparous and mul- } \\
\text { tiparous women with } \\
\text { a singleton preg- } \\
\text { nancy at } \geq 37 \mathrm{wk} \\
\text { gestation } \\
\text { - Bishop's score }<6 \\
\text { - Cervical dilation } \\
\leq 2 \mathrm{~cm}\end{array}$ & $\begin{array}{l}\text { Primary: } \\
\text { - Time to delivery } \\
\text { - Secondary: } \\
\text { - Cesarean delivery rate } \\
\text { - Time to vaginal delivery } \\
\text { - Time to delivery cen- } \\
\text { sored for cesarean sec- } \\
\text { tion } \\
\text { - Time to active labor } \\
\text { - Delivery } \leq 12 \mathrm{~h} \\
\text { - Delivery } \leq 24 \mathrm{~h} \\
\text { - Maternal length of stay } \\
\text { - Indication for cesarean } \\
\text { section } \\
\text { - Maternal morbidity } \\
\text { composite ( } \geq 1 \text { of third } \\
\text { or fourth degree perineal } \\
\text { laceration, blood trans- } \\
\text { fusion, endometritis, } \\
\text { wound separation infec- } \\
\text { tion, venous throm- } \\
\text { boembolism, hysterec- } \\
\text { tomy, ICU admission, } \\
\text { death) } \\
\text { - Chorioamnionitis } \\
\text { - Use of terbutaline } \\
\text { - Intrauterine pressure } \\
\text { catheter } \\
\text { - Amnioinfusion }\end{array}$ & $\begin{array}{l}\text { - Median time to deliv- } \\
\text { ery was faster with } \\
\text { combination methods } \\
\text { vs. single agents } \\
\text { (Foley's plus miso- } \\
\text { prostol, } 13.1 \text { h; Foley's } \\
\text { plus oxytocin, } 14.5 ; \\
\text { misoprostol, } 17.6 \text { h; } \\
\text { Foley's, } 17.7 \text { h; } \\
\text { p }<0.001 \text { ) } \\
\text { - After censoring for } \\
\text { cesarean delivery and } \\
\text { adjusting for parity, } \\
\text { women who received } \\
\text { Foley's plus misopros- } \\
\text { tol were almost twice } \\
\text { as likely to deliver } \\
\text { before those receiving } \\
\text { misoprostol or Foley's } \\
\text { alone (hazard ratio } \\
=1.92 \text { and } 1.87, \\
\text { respectively) } \\
\text { - No significant differ- } \\
\text { ences in cesarean } \\
\text { delivery rates, indica- } \\
\text { tion for cesarean } \\
\text { delivery, composite } \\
\text { maternal outcome, or } \\
\text { neonatal outcomes }\end{array}$ \\
\hline
\end{tabular}


Table 1 (Continued)

\begin{tabular}{|c|c|c|c|c|c|}
\hline Clinical trial & Drug/device & Trial design & Trial population & Outcomes & Main results \\
\hline & & & & $\begin{array}{l}\text { - Analgesia use } \\
\text { - Neonatal morbidity } \\
\text { composite ( } \geq 1 \text { of severe } \\
\text { respiratory distress syn- } \\
\text { drome, sepsis, blood } \\
\text { transfusion, hypoxic- } \\
\text { ischemic encephalopa- } \\
\text { thy, intraventricular } \\
\text { hemorrhage grade } 3 \text { or } \\
4, \text { NEC, receipt of head } \\
\text { cooling) } \\
\text { - NICU admission and } \\
\text { length of stay }\end{array}$ & \\
\hline $\begin{array}{l}\text { Edwards et al, Foley } \\
\text { catheter compared with } \\
\text { the controlled-release } \\
\text { dinoprostone insert: a } \\
\text { randomized controlled } \\
\text { trial }^{39}\end{array}$ & $\begin{array}{l}\text { Foley's catheter vs. } \\
\text { vaginal dinoprostone } \\
\text { insert } \\
\text { Dosing: } \\
\text { - } 16 \mathrm{~F} \text { Foley's catheter } \\
\text { inflated to } 30 \mathrm{~mL} \\
\text { - } 10 \mathrm{mg} \text { vaginal dino- } \\
\text { prostone insert, max } \\
12 \mathrm{~h}\end{array}$ & Multicenter RCT & $\begin{array}{l}n=376 \\
\text { - Nulliparous and mul- } \\
\text { tiparous women with } \\
\text { a singleton preg- } \\
\text { nancy at } \geq 36 \mathrm{wk} \\
\text { gestation } \\
\text { - Cervical dilation } \\
<3 \mathrm{~cm} \text {; if } 2 \mathrm{~cm} \\
\text { dilated, }<80 \% \text { effaced }\end{array}$ & $\begin{array}{l}\text { Primary: } \\
\text { - Time to delivery } \\
\text { - Secondary: } \\
\text { - Delivery } \leq 12 \mathrm{~h} \\
\text { - Delivery } \leq 24 \mathrm{~h} \\
\text { - Vaginal delivery } \leq 24 \mathrm{~h} \\
\text { - Tachysystole } \\
\text { - Chorioamnionitis } \\
\text { - Endometritis } \\
\text { - Other postpartum com- } \\
\text { plications (e.g., pneumo- } \\
\text { nia, venous } \\
\text { thromboembolism, ICU } \\
\text { admission, maternal } \\
\text { death) } \\
\text { - Cesarean delivery rate } \\
\text { - Early neonatal outcomes } \\
\text { (e.g., weight, } 1 \text { and 5-min } \\
\text { Apgar's scores, arterial } \\
\text { cord pH, NICU admission) }\end{array}$ & $\begin{array}{l}\text { - Women in the Foley's } \\
\text { catheter group had a } \\
\text { shorter median time to } \\
\text { delivery ( } 21.6 \text { vs. } \\
26.6 \mathrm{~h} ; p=0.003 \text { ) and } \\
\text { vaginal delivery (20.1 } \\
\text { vs. } 24.3 \mathrm{~h} ; p=0.005) \\
\text { vs. women who were } \\
\text { treated with dinopros- } \\
\text { tone } \\
\text { - More women who } \\
\text { received the Foley's } \\
\text { catheter delivered } \\
\leq 24 \mathrm{~h}(56 \text { vs. } 40 \% \text {; } \\
p=0.003 \text { ) and deliv- } \\
\text { ered vaginally } \leq 24 \mathrm{~h} \\
\text { (44 vs. } 30 \% ; p=0.004 \text { ) } \\
\text { - No significant differ- } \\
\text { ences in other secondary } \\
\text { outcomes }\end{array}$ \\
\hline $\begin{array}{l}\text { Suffecool et al, labor } \\
\text { induction in nulliparous } \\
\text { women with an unfa- } \\
\text { vorable cervix: double } \\
\text { balloon catheter versus } \\
\text { dinoprostone }\end{array}$ & $\begin{array}{l}\text { Vaginal dinoprostone } \\
\text { insert vs. double-bal- } \\
\text { loon catheter } \\
\text { Dosing: } \\
\text { - Both balloons of the } \\
\text { double-balloon } \\
\text { catheter inflated to } \\
80 \mathrm{~mL} \\
\text { - } 10 \mathrm{mg} \text { vaginal dino- } \\
\text { prostone insert, max } \\
12 \mathrm{~h}\end{array}$ & Single-center RCT & $\begin{array}{l}n=62 \\
\text { - Nulliparous women } \\
\text { with a singleton } \\
\text { pregnancy at } \\
\geq 37 \text { wk gestation } \\
\text { - Bishop's score }<6\end{array}$ & $\begin{array}{l}\text { Primary: } \\
\text { - Time to delivery } \\
\text { Secondary: } \\
\text { - Delivery } \leq 24 \mathrm{~h} \\
\text { - Cesarean delivery rate } \\
\text { - Time to active labor } \\
\text { - Operative vaginal } \\
\text { delivery } \\
\text { - Maternal or fetal } \\
\text { adverse events (e.g., } \\
\text { tachysystole) }\end{array}$ & $\begin{array}{l}\text { - Women who received } \\
\text { the double-balloon } \\
\text { catheter had a shorter } \\
\text { mean time to delivery } \\
(17.9 \text { vs. } 26.3 \mathrm{~h} \text {; } \\
p=0.0001) \text { and mean } \\
\text { time to vaginal deliv- } \\
\text { ery }(19.1 \text { vs. } 24.4 \mathrm{~h} \text {; } \\
p=0.05) \text { vs. those } \\
\text { who received vaginal } \\
\text { dinoprostone } \\
\text { More women in the } \\
\text { double-balloon group } \\
\text { delivered } \leq 24 \text { h ( } 87 \\
\text { vs. } 48 \% ; p=0.002) \\
\text { Cesarean delivery rates } \\
\text { were similar in both } \\
\text { treatment groups }\end{array}$ \\
\hline $\begin{array}{l}\text { Carbone et al, combina- } \\
\text { tion of Foley bulb and } \\
\text { vaginal misoprostol } \\
\text { compared with vaginal } \\
\text { misoprostol alone for } \\
\text { cervical ripening and } \\
\text { labor induction: a ran- } \\
\text { domized controlled } \\
\text { trial }^{49}\end{array}$ & $\begin{array}{l}\text { Foley's catheter plus } \\
\text { vaginal misoprostol } \\
\text { tablet vs. vaginal miso- } \\
\text { prostol tablet alone } \\
\text { Dosing: } \\
\text { - Foley's catheter } \\
\text { inflated to } 60 \mathrm{~mL} \\
\text { - } 25 \mu \mathrm{g} \text { vaginal miso- } \\
\text { prostol tablet given } \\
\text { every } 4 \mathrm{~h}\end{array}$ & Single-center RCT & $\begin{array}{l}n=123 \\
\text { - Nulliparous women } \\
\text { with a singleton } \\
\text { pregnancy at } \\
\geq 24 \text { wk gestation } \\
\text { - Bishop's score }<6\end{array}$ & $\begin{array}{l}\text { Primary: } \\
\text { - Time to delivery } \\
\text { Secondary: } \\
\text { - Mode of delivery } \\
\text { - Tachysystole with fetal } \\
\text { heart rate decelera- } \\
\text { tions requiring terbuta- } \\
\text { line use } \\
\text { - Postpartum hemor- } \\
\text { rhage } \\
\text { - Chorioamnionitis } \\
\text { - Apgar's scores } \\
\text { - NICU admission }\end{array}$ & $\begin{array}{l}\text { - Women who received } \\
\text { Foley's plus misopros- } \\
\text { tol had a shorter mean } \\
\text { time to delivery vs. } \\
\text { misoprostol alone } \\
(15.3 \text { vs. } 18.3 \mathrm{~h} ; \\
p=0.03) \\
\text { - There were no signifi- } \\
\text { cant differences in } \\
\text { mode of delivery, labor } \\
\text { complications, or } \\
\text { adverse maternal and } \\
\text { neonatal outcomes }\end{array}$ \\
\hline $\begin{array}{l}\text { Wing et al (2013), mis- } \\
\text { oprostol vaginal insert } \\
\text { and time to vaginal } \\
\text { delivery: a randomized } \\
\text { controlled trial }^{54}\end{array}$ & $\begin{array}{l}\text { Vaginal misoprostol } \\
\text { insert vs. vaginal dino- } \\
\text { prostone insert } \\
\text { Dosing: } \\
\text { - } 200 \mu \text { gaginal miso- } \\
\text { prostol insert, max } \\
24 \mathrm{~h} \\
\text { - } 10 \mathrm{mg} \text { vaginal dino- } \\
\text { prostone insert, max } \\
24 \mathrm{~h}\end{array}$ & $\begin{array}{l}\text { Double-blind multicen- } \\
\text { ter RCT }\end{array}$ & $\begin{array}{l}n=1,358 \\
\text { - Nulliparous and mul- } \\
\text { tiparous women with } \\
\text { a singleton preg- } \\
\text { nancy at } \geq 36 \mathrm{wk} \\
\text { gestation } \\
\text { - Bishop's score }<4 \\
\text { - BMl } \leq 50 \mathrm{~kg} / \mathrm{m}^{2} \\
\text { - Parity } \leq 3\end{array}$ & $\begin{array}{l}\text { Primary: } \\
\text { - Time to vaginal delivery } \\
\text { - Cesarean delivery rate } \\
\text { Secondary: } \\
\text { - Time to delivery } \\
\text { - Time to active labor } \\
\text { - Requirement for pre- } \\
\text { delivery oxytocin } \\
\text { - Vaginal or any delivery } \\
\leq 12 \text { and } \leq 24 \mathrm{~h} \\
\text { - Maternal or fetal } \\
\text { adverse events (e.g., } \\
\text { tachysystole) }\end{array}$ & $\begin{array}{l}\text { - Compared with } \\
\text { the dinoprostone } \\
\text { insert, women } \\
\text { who received the } \\
\text { misoprostol insert } \\
\text { had a shorter median } \\
\text { time to vaginal } \\
\text { delivery ( } 32.8 \text { vs. } \\
21.5 \text { h), any delivery } \\
\text { ( } 27.3 \text { vs. } 18.3 \text { h), } \\
\text { and onset to active } \\
\text { labor ( } 18.6 \text { vs. } 12.1 \mathrm{~h} \text {; } \\
\text { p< } 0.001 \text { for all } \\
\text { comparisons) }\end{array}$ \\
\hline
\end{tabular}


Table 1 (Continued)

\begin{tabular}{|c|c|c|c|c|c|}
\hline Clinical trial & Drug/device & Trial design & Trial population & Outcomes & Main results \\
\hline & & & & & $\begin{array}{l}\text { - Cesarean delivery } \\
\text { rates were similar with } \\
\text { both treatments } \\
\text { - Tachysystole requiring } \\
\text { intervention occurred } \\
\text { in } 13.3 \% \text { of women } \\
\text { receiving misoprostol } \\
\text { and } 4.0 \% \text { of women } \\
\text { receiving dinopros- } \\
\text { tone }(p<0.001)\end{array}$ \\
\hline $\begin{array}{l}\text { Fitzpatrick et al, cervical } \\
\text { ripening with Foley bal- } \\
\text { loon plus fixed vs. incre- } \\
\text { mental low-dose } \\
\text { oxytocin: a randomized } \\
\text { controlled trial }^{58}\end{array}$ & $\begin{array}{l}\text { Foley's catheter plus } \\
\text { fixed low-dose oxytocin } \\
\text { infusion vs. Foley's } \\
\text { catheter plus standard } \\
\text { incremental low-dose } \\
\text { oxytocin infusion } \\
\text { Dosing: } \\
\text { - Fixed low-dose oxy- } \\
\text { tocin infusion of } \\
2 \mathrm{mU} / \mathrm{min} \\
\text { - Incremental low- } \\
\text { dose oxytocin infu- } \\
\text { sion starting at } 1 \mathrm{mU} / \\
\text { min and increasing by } \\
2 \mathrm{mU} / \mathrm{min} \text { every } \\
30 \mathrm{~min} \text {, max dose } \\
20 \mathrm{mU} / \mathrm{min}\end{array}$ & Single-center RCT & $\begin{array}{l}n=116 \\
\text { - Nulliparous and mul- } \\
\text { tiparous women with } \\
\text { a singleton preg- } \\
\text { nancy at } \geq 37 \text { wk } \\
\text { gestation } \\
\text { - Bishop's score }<6\end{array}$ & $\begin{array}{l}\text { Primary: } \\
\text { - Time to delivery } \\
\text { Secondary: } \\
\text { - Time to Foley's expul- } \\
\text { sion } \\
\text { - Time to active labor } \\
\text { - Time to second stage } \\
\text { - Cesarean delivery rate } \\
\text { - Tachysystole }\end{array}$ & $\begin{array}{l}\text { - There were no signifi- } \\
\text { cant differences in } \\
\text { median time to deliv- } \\
\text { ery in women who } \\
\text { received the Foley's } \\
\text { catheter plus fixed low- } \\
\text { dose or incremental } \\
\text { low-dose oxytocin } \\
(23.7 \text { vs. } 19.2 \mathrm{~h} \text {; } \\
p=0.388) \\
\text { - No significant differ- } \\
\text { ences in other second- } \\
\text { ary outcomes }\end{array}$ \\
\hline $\begin{array}{l}\text { Hill et al, a randomized } \\
\text { clinical trial comparing } \\
\text { vaginal misoprostol ver- } \\
\text { sus cervical Foley plus } \\
\text { oral misoprostol for cer- } \\
\text { vical ripening and labor } \\
\text { induction } 72\end{array}$ & $\begin{array}{l}\text { Foley's catheter plus } \\
\text { oral misoprostol vs. } \\
\text { vaginal misoprostol } \\
\text { Dosing: } \\
\text { - } 24 \mathrm{~F} \text { Foley's catheter } \\
\text { inflated to } 50 \mathrm{~mL} \\
\text { - } 100 \mu \mathrm{g} \text { oral miso- } \\
\text { prostol at } 4 \text { to } 6-\mathrm{h} \\
\text { intervals, max } 4 \\
\text { doses } \\
\text { - Initial dose of } 25 \mu \mathrm{g} \\
\text { vaginal misoprostol } \\
\text { followed by } 50 \mu \mathrm{g} \\
\text { doses at } 3 \text { to } 6-\mathrm{h} \\
\text { intervals, max } 8 \\
\text { doses }\end{array}$ & Single-center RCT & $\begin{array}{l}n=126 \\
\text { - Nulliparous and mul- } \\
\text { tiparous women with } \\
\text { a singleton preg- } \\
\text { nancy at } \geq 24 \text { wk } \\
\text { gestation } \\
\text { - Bishop's score } \leq 4\end{array}$ & $\begin{array}{l}\text { Primary: } \\
\text { - Time to delivery } \\
\text { Secondary: } \\
\text { - Delivery } \leq 24 \mathrm{~h} \\
\text { - Time from rupture of } \\
\text { membranes to delivery } \\
\text { - Requirement for oxyto- } \\
\text { cin } \\
\text { - Time to second stage }\end{array}$ & $\begin{array}{l}\text { - Women who received } \\
\text { Foley's plus oral miso- } \\
\text { prostol had a shorter } \\
\text { time to delivery (12.9 } \\
\text { vs. } 17.8 \text { h; } p<0.001) \\
\text { and were more likely to } \\
\text { deliver } \leq 24 \text { h (rela- } \\
\text { tive risk }=1.36) \text { vs. } \\
\text { those who received } \\
\text { vaginal misoprostol } \\
\text { - Cesarean delivery rate } \\
\text { and indications for } \\
\text { cesarean delivery were } \\
\text { similar in both treat- } \\
\text { ment groups } \\
\text { - A higher rate of tachy- } \\
\text { systole was seen with } \\
\text { Foley's plus oral miso- } \\
\text { prostol vs. vaginal } \\
\text { misoprostol (39 vs. } \\
21 \% ; p=0.015) \\
\text { - No significant differ- } \\
\text { ences in neonatal } \\
\text { outcomes }\end{array}$ \\
\hline $\begin{array}{l}\text { Fonseca et al, rando- } \\
\text { mized trial of preinduc- } \\
\text { tion cervical ripening: } \\
\text { misoprostol vs } \\
\text { oxytocin }^{79}\end{array}$ & $\begin{array}{l}\text { Vaginal misoprostol vs. } \\
\text { oxytocin infusion } \\
\text { Dosing: } \\
\text { - } 25 \mu \text { gaginal miso- } \\
\text { prostol every } 4 \mathrm{~h} \\
\text { - Oxytocin infusion } \\
\text { started at } 4 \mathrm{mU} / \mathrm{min} \\
\text { for nulliparous } \\
\text { women and } 2 \mathrm{mU} / \\
\text { min for multiparous } \\
\text { women; increased by } \\
4 \mathrm{mu} / \mathrm{min} \text { or } 2 \mathrm{mU} / \\
\text { min, respectively, } \\
\text { every } 15 \mathrm{~min}, \mathrm{max} \\
\text { dose } 40 \mathrm{mU} / \mathrm{min}\end{array}$ & Single-center RCT & $\begin{array}{l}n=361 \\
\text { - Nulliparous and mul- } \\
\text { tiparous women with } \\
\text { a singleton preg- } \\
\text { nancy at } \geq 24 \text { wk } \\
\text { gestation } \\
\text { - Bishop's score }<5\end{array}$ & $\begin{array}{l}\text { Primary: } \\
\text { - Vaginal delivery rate } \\
\text { Secondary: } \\
\text { - Time to delivery } \\
\text { - Time to active labor } \\
\text { - Duration of labor and } \\
\text { delivery } \\
\text { - Neonatal outcomes } \\
\text { (birth weight, } 1 \text { and } \\
\text { 5-min Apgar's scores, } \\
\text { umbilical artery cord } \\
\text { blood pH and gases, } \\
\text { and NICU admission) }\end{array}$ & $\begin{array}{l}\text { - Vaginal delivery rates } \\
\text { were similar in women } \\
\text { treated with miso- } \\
\text { prostol or oxytocin ( } 81 \\
\text { vs. } 87 \% ; p=0.31 \text { ) } \\
\text { - Mean time to delivery } \\
\text { was shorter with oxy- } \\
\text { tocin vs. misoprostol } \\
(13.1 \text { vs. } 16.3 \mathrm{~h} \text {; } \\
p=0.005) \\
\text { - No significant differ- } \\
\text { ences in neonatal } \\
\text { outcomes }\end{array}$ \\
\hline $\begin{array}{l}\text { Pettker et al, transcervi- } \\
\text { cal Foley catheter with } \\
\text { and without oxytocin for } \\
\text { cervical ripening: a ran- } \\
\text { domized controlled } \\
\text { trial }^{81}\end{array}$ & $\begin{array}{l}\text { Foley's catheter plus } \\
\text { low-dose oxytocin infu- } \\
\text { sion vs. Foley's catheter } \\
\text { alone } \\
\text { Dosing: } \\
\text { - } 20 \text { F Foley's catheter } \\
\text { inflated to } 30 \mathrm{~mL} \\
\text { - Oxytocin infusion } \\
\text { started at } 1 \mathrm{mU} / \mathrm{min} \\
\text { and increased by } \\
1 \mathrm{mU} / \mathrm{min} \text { every } \\
15 \mathrm{~min}, \mathrm{max} \\
10 \mathrm{mU} / \mathrm{min}\end{array}$ & Single-center RCT & $\begin{array}{l}n=183 \\
\text { - Nulliparous and mul- } \\
\text { tiparous women with } \\
\text { a singleton preg- } \\
\text { nancy at }>23 \text { wk } \\
\text { gestation } \\
\text { - Women with only } 1 \\
\text { previous cesarean } \\
\text { delivery were not } \\
\text { excluded }\end{array}$ & $\begin{array}{l}\text { Primary: } \\
\text { - Delivery } \leq 24 \mathrm{~h} \\
\text { Secondary: } \\
\text { - Vaginal delivery } \leq 24 \mathrm{~h} \\
\text { - Vaginal delivery rate } \\
\text { - Duration of ripening } \\
\text { - Time to delivery } \\
\text { - Chorioamnionitis } \\
\text { - Hemorrhage } \\
\text { - Analgesia use }\end{array}$ & $\begin{array}{l}\text { - There were no signifi- } \\
\text { cant differences in the } \\
\text { proportion of overall } \\
\text { ( } 65 \text { vs. } 60 \% ; p=0.50 \text { ) } \\
\text { and vaginal ( } 48 \text { vs. } \\
46 \% ; p=0.82 \text { ) deliv- } \\
\text { eries } \leq 24 \text { h in women } \\
\text { who received Foley's } \\
\text { plus oxytocin and } \\
\text { those who received } \\
\text { the Foley's alone }\end{array}$ \\
\hline
\end{tabular}


Table 1 (Continued)

\begin{tabular}{|c|c|c|c|c|c|}
\hline Clinical trial & Drug/device & Trial design & Trial population & Outcomes & Main results \\
\hline & & & & & $\begin{array}{l}\text { - No significant differ- } \\
\text { ences in cesarean } \\
\text { delivery rates or rates } \\
\text { of maternal complica- } \\
\text { tions } \\
\text { - Women in the Foley's } \\
\text { plus oxytocin group } \\
\text { required more regio- } \\
\text { nal analgesia during } \\
\text { induction ( } 23 \text { vs. 9\%; } \\
p=0.01 \text { ) }\end{array}$ \\
\hline $\begin{array}{l}\text { Wing (2008), misopros- } \\
\text { tol vaginal insert com- } \\
\text { pared with } \\
\text { dinoprostone vaginal } \\
\text { insert: a randomized } \\
\text { controlled trial }^{84}\end{array}$ & $\begin{array}{l}\text { Vaginal misoprostol } \\
\text { insert vs. vaginal dino- } \\
\text { prostone insert } \\
\text { Dosing: } \\
\text { - } 50 \mu \mathrm{g} \text { or } 100 \mu \mathrm{g} \\
\text { vaginal misoprostol } \\
\text { insert, max } 24 \mathrm{~h} \\
\text { - } 10 \mathrm{mg} \text { vaginal dino- } \\
\text { prostone insert, max } \\
24 \mathrm{~h}\end{array}$ & $\begin{array}{l}\text { Double-blind multicen- } \\
\text { ter RCT }\end{array}$ & $\begin{array}{l}n=1,308 \\
\text { - Nulliparous and mul- } \\
\text { tiparous women with } \\
\text { a singleton preg- } \\
\text { nancy at } \geq 36 \text { wk } \\
\text { gestation } \\
\text { - Bishop's score }<4 \\
\text { - Parity } \leq 3\end{array}$ & $\begin{array}{l}\text { Primary: } \\
\text { - Time to vaginal delivery } \\
\text { - Cesarean delivery rate } \\
\text { Secondary: } \\
\text { - Composite modified } \\
\text { Bishop's score at } 12 \mathrm{~h} \\
\text { (vaginal delivery } \\
\leq 12 \mathrm{~h} \text {, increase in } \\
\text { Bishop's score } \geq 3 \text { from } \\
\text { baseline, Bishop's score } \\
\geq 6 \\
\text { - Delivery } \leq 12 \text { and } \\
\leq 24 \text { h } \\
\text { - Predelivery oxytocin } \\
\text { administration } \\
\text { - Time to active labor }\end{array}$ & $\begin{array}{l}\text { - Median time to vaginal } \\
\text { delivery was similar in } \\
\text { women treated with } \\
\text { the misoprostol } 100 \\
\mu \mathrm{g} \text { vaginal insert and } \\
\text { the dinoprostone } \\
\text { insert ( } 1,596 \text { vs. } \\
1,650 \text { min; } p=0.97) \\
\text { - Women in the miso- } \\
\text { prostol } 50 \mu \text { group } \\
\text { had a longer time to } \\
\text { vaginal delivery vs. } \\
\text { those treated with } \\
\text { dinoprostone ( } 2,127 \\
\text { vs. } 1,650 \text { min; } \\
p=0.01) \\
\text { - No significant differ- } \\
\text { ences in cesarean } \\
\text { delivery rates between } \\
\text { either misoprostol } \\
\text { dose and dinoprostone } \\
\text { - All three treatments } \\
\text { had similar safety } \\
\text { profiles }\end{array}$ \\
\hline
\end{tabular}

Abbreviations: BMI, body mass index; ICU, intensive care unit; NEC, necrotizing enterocolitis; NICU, neonatal intensive care unit; pPROM, preterm premature rupture of membranes; RCT, randomized controlled trial.

Table 2 Summary of limitations in published labor induction clinical trials (all RCTs) $23-85$

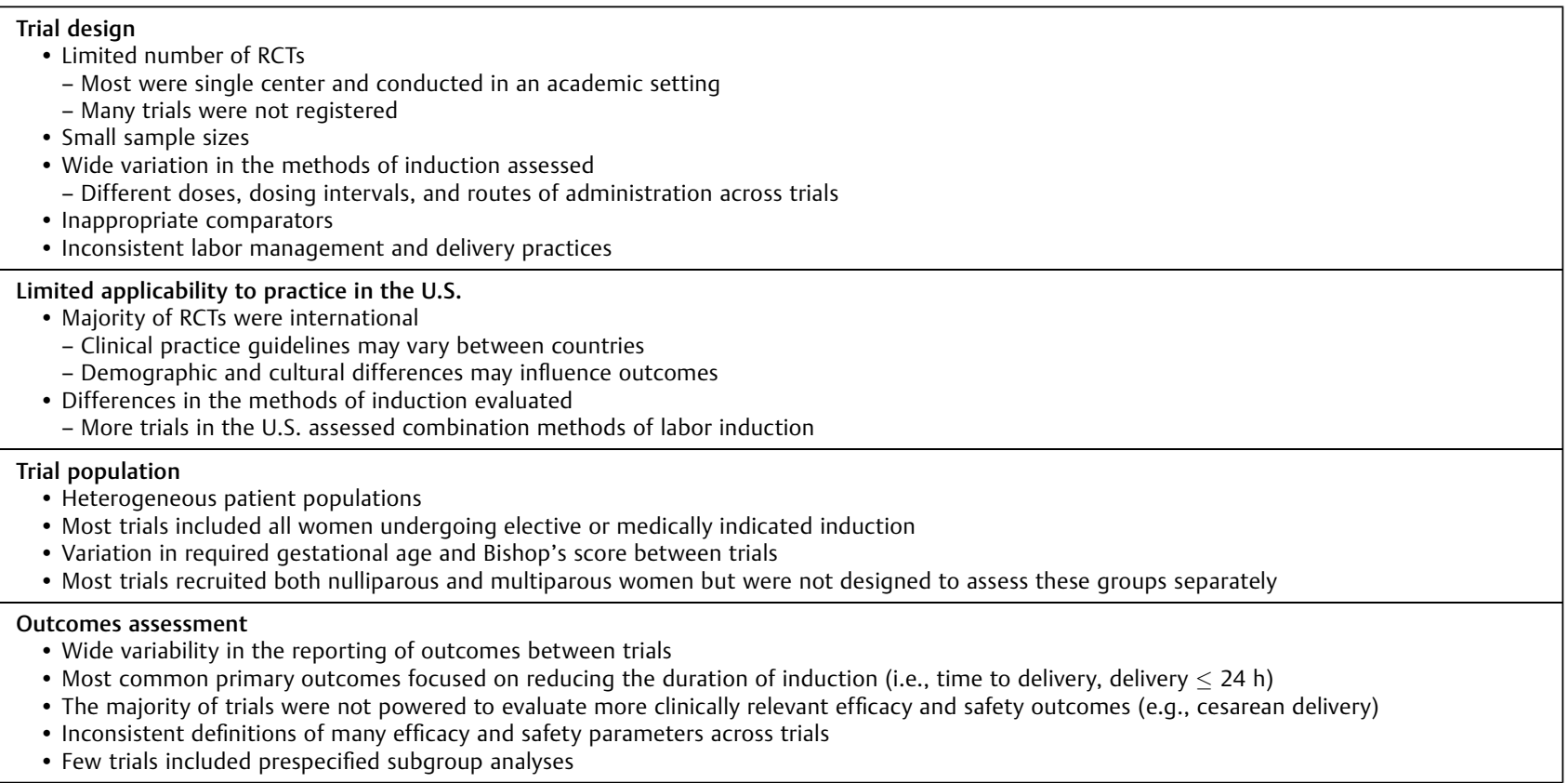

Abbreviation: RCT, randomized controlled trial. 
generalizability of the results to other community-based populations. Among the 12 multicenter trials, the number of centers ranged from 2 to 49 and tended to include both university-affiliated and community hospitals.

Sample size estimation and power analysis are crucial components of any well-designed trial. A sufficiently large sample is needed for reliable accurate results; however, sample size was limited in nearly all trials included in our search. Although power-based sample size calculations were used in 53 RCTs, most trials were underpowered to detect significant differences in secondary efficacy and safety outcomes. In addition to sample size, proper randomization is another important aspect of clinical trial design used to balance treatment groups and eliminate selection bias. Most of the RCTs $(n=58)$ included in our search described the method of randomization, with the majority using simple randomization techniques (e.g., computergenerated random numbers). Few RCTs included in our search were blinded. However, in most cases, it was not possible to blind patients and medical staff due to the nature of the intervention (e.g., Foley's catheter vs. vaginal dinoprostone).

\section{Trial Design: Treatment Protocols}

There was variation in the methods (pharmacological, mechanical, or both) and protocols for labor induction among the RCTs included in our search. Pharmacological interventions varied by dose, dose intervals, and route of administration. For example, misoprostol was administered through sublingual, oral, or vaginal routes. Most trials used 25 or $50 \mu \mathrm{g}$ misoprostol tablets administered every 3 to 6 hours; two trials used a 50, 100, or $200 \mu$ g controlled-release misoprostol vaginal insert. ${ }^{54,84}$ Oral misoprostol was given as a static dose (tablet or solution) or titrated solution. Dinoprostone was typically administered as a $10 \mathrm{mg}$ controlled-release vaginal insert kept in place for 12 to 24 hours. Some trials used dinoprostone gel or tablets administered vaginally (ranging from $1-3 \mathrm{mg}$ every $4-8$ hours) $36,43,64,66,71,74,77,82$ or dinoprostone gel administered intracervically ( $0.5 \mathrm{mg}$ every 6 or 12 hours). ${ }^{43,56,68,80}$ Protocols for oxytocin infusion varied, with starting doses ranging from 1 to $4 \mathrm{mU} / \mathrm{min}$, rate increases every 15 to 30 minutes, and maximum dosing ranging from 10 to $40 \mathrm{mU} / \mathrm{min}$. Among the trials that used mechanical methods for induction, most used the Foley catheter $(n=23)$; different sizes of catheters were used (e.g., 16F, 18F) and were inflated with varying volumes of water or saline solution (30-60 mL). Doubleballoon catheters were used in 11 trials and each balloon was filled with up to $80 \mathrm{~mL}$ of fluid. The variation in doses and routes of administration with pharmacological agents, as well as the different types of balloon catheters and inflation volumes used in the labor induction trials can lead to different outcomes, making it difficult to compare methods across trials. It should be noted that findings from a recent meta-analysis of six trials suggest there are no differences in labor induction outcomes (i.e., time to delivery, vaginal and cesarean delivery rates, maternal satisfaction) with single versus double balloon catheters. ${ }^{86}$
It is also difficult to compare treatments within some trials due to the use of inappropriate comparators. Comparing methods with different mechanisms of action and efficacy/safety profiles complicate results and makes it difficult to interpret the data. For example, two trials compared the Foley catheter plus oxytocin versus vaginal misoprostol. ${ }^{28,70}$ Another compared vaginal dinoprostone with or without sequential oxytocin versus vaginal misoprostol plus sequential oxytocin. ${ }^{83}$ These trials may have yielded more meaningful data if the combination agents were compared with the same single agents (e.g., Foley's catheter plus oxytocin vs. Foley's catheter alone or oxytocin alone).

\section{Trial Design: Labor Management}

Differences in labor management following labor induction also varied between the trials included in our search. In some trials, oxytocin administration was permitted if a patient did not go into active labor but explicit guidelines were typically not provided. Active labor and delivery, including the use of oxytocin for labor augmentation, were managed according to standard institutional practices. For example, a trial comparing the Foley catheter with or without oxytocin used a lowdose oxytocin regimen for induction and augmentation that was specific to that institution. ${ }^{81}$ This trial found that the addition of oxytocin to the Foley catheter did not shorten the time to delivery. However, subsequent studies that used a higher dose of oxytocin per institutional protocol reported a shorter time to delivery with the combination method. ${ }^{25,27}$ Other differences in protocols (e.g., inflation of the Foley balloon to $30 \mathrm{~mL}$ vs. $60 \mathrm{~mL}$ ) and patient populations likely contributed to the differences in study outcomes. However, differences in labor management protocols across trials or within some multicenter trials may also influence the outcomes of labor induction studies, making it more difficult to compare treatment methods.

\section{Applicability to Clinical Practice in the U.S.: Clinical Trial Location}

Most RCTs identified in our search were international, with the majority conducted in South Asian $(n=15)$, West Asian $(n=11)$, European $(n=10)$, and African $(n=8)$ countries. Just 12 of the 63 RCTs were conducted within the U.S. (-Table 1). Results from international and U.S. trials should be interpreted with caution because clinical practice guidelines in other countries (e.g., WHO, Society of Obstetricians and Gynaecologists of Canada [SOGC], Royal College of Obstetricians and Gynaecologists [RCOG], National Institute of Health and Care Excellence [NICE]) can differ from those in the U.S. where ACOG recommendations determine standard of care. For example, WHO and ACOG recommend the use of misoprostol or dinoprostone for induction of labor, ${ }^{2,4}$ while NICE guidelines prefer dinoprostone and recommend misoprostol only for women with intrauterine fetal death or in the context of a clinical trial. ${ }^{87}$ Although guidelines may recommend the use of a particular agent, other agents may be preferred due to availability or physician preference and experience. ${ }^{88,89}$ WHO guidelines, which are intended for a global audience, recommend the use of misoprostol or 
dinoprostone as first-line induction agents, ${ }^{4}$ yet oxytocin alone is the preferred method in many Latin American, African, and Asian countries. ${ }^{88,90}$ It is likely that demographic and cultural differences also influence treatment; the potential impact of these differences on trial outcomes is discussed in the "Trial population" section of this review.

\section{Applicability to Clinical Practice in the U.S.: Use of Combination Methods}

More than half of the clinical trials conducted in the U.S. (58\%; $n=7 / 12$ ) evaluated combination methods of labor induction compared with just $22 \%$ of the international trials ( $n=11 / 51$ ). The trials in the U.S. assessed the Foley catheter in combination with oxytocin or misoprostol versus various comparators. The Foley catheter plus oxytocin was compared with the Foley catheter alone $(n=1),{ }^{81}$ sequential use of the Foley catheter and oxytocin $(n=2),{ }^{25,27}$ or a different oxytocin dosing protocol (i.e., fixed vs. incremental low-dose oxytocin; $n=1){ }^{58}$ The Foley catheter plus vaginal misoprostol was compared with vaginal misoprostol alone $(n=1) ; 49$ another clinical trial compared the Foley catheter plus oral misoprostol versus vaginal misoprostol alone. ${ }^{72} \mathrm{~A}$ separate trial compared four different treatment groups: the Foley catheter plus oxytocin versus the Foley catheter plus vaginal misoprostol versus the Foley catheter alone versus vaginal misoprostol alone. ${ }^{29}$ Despite the differences in trial design, all but one of the trials found that the combination method was more effective in inducing labor; the primary efficacy outcomes reported in these trials were time to delivery $(n=5)$ or delivery within 24 hours $(n=2)$. No significant differences in maternal complications or adverse neonatal outcomes between the combination methods and comparators were reported in any of the trials.

The international trials assessed a wider variety of combination methods, with the majority evaluating mechanical methods plus oxytocin or misoprostol: the Foley catheter plus oxytocin versus vaginal misoprostol $(n=2),{ }^{28,70}$ the Foley catheter plus vaginal misoprostol versus vaginal misoprostol or the Foley catheter alone $(n=3),{ }^{26,41,53}$ and double-balloon catheter plus oral misoprostol versus oral misoprostol alone $(n=2) .{ }^{35,65}$ A few of the international trials assessed combinations of pharmacological methods, including the dinoprostone vaginal insert plus concurrent oxytocin versus sequential use of the dinoprostone insert and oxytocin $(n=1),{ }^{63}$ the dinoprostone vaginal insert with or without sequential oxytocin versus vaginal misoprostol plus sequential oxytocin $(n=1),{ }^{83}$ and vaginal misoprostol plus sequential oxytocin versus oxytocin alone $(n=2) .{ }^{62,69}$ The primary outcomes reported in the international combination method trials included time to delivery $(n=8)$ and vaginal delivery within 24 hours $(n=1)$ or 48 hours $(n=2)$. Just more than half the trials $(n=6 / 11)$ reported an improvement in time to vaginal delivery or vaginal delivery within 24 or 48 hours with the combination methods; however, two trials were not adequately powered. There were no significant differences in maternal complications or neonatal outcomes, except for a higher number of infants with 5-minute Apgar's scores $<7$ with the double-balloon catheter plus sequential oral misoprostol versus oral misoprostol alone ( 8 vs. $1 ; p=0.04),{ }^{35}$ and lower incidences of uterine hyperstimulation ( 8 vs. $40 \% ; p<0.001$ ) and meconium-stained liquor ( 6 vs. $27 \%$; $p=0.001$ ) with the Foley catheter plus vaginal misoprostol versus vaginal misoprostol alone. $^{41}$

It is unclear why combination methods (i.e., the Foley catheter plus oxytocin or misoprostol) were used more often in trials conducted within the U.S. As described previously, the use of oxytocin alone is the preferred method of labor induction in many regions outside the U.S., ${ }^{88-90}$ thus differences in the frequency and types of combination methods used in the U.S. and international trials may be reflective of different demographics and cultural preferences among clinical trial populations.

\section{Applicability to Clinical Practice in the U.S.: Use of Misoprostol}

The majority of the international trials (75\%; $n=38 / 51$ ) included misoprostol as a comparator compared with 6 of the 12 trials conducted in the U.S. Most of the international trials assessed different routes of administration (e.g., oral vs. vaginal) or compared misoprostol with dinoprostone. Among the international trials that assessed misoprostol versus dinoprostone, nearly all compared vaginal misoprostol with vaginal dinoprostone gel or the vaginal dinoprostone insert; three trials compared vaginal misoprostol with intracervical dinoprostone gel. In most of these trials, misoprostol was associated with improved efficacy outcomes compared with dinoprostone, including a shorter time to delivery, more vaginal deliveries $\leq 12$ or $\leq 24$ hours, improved Bishop's scores, and less requirement for oxytocin. ${ }^{44,56,66,68,73,80}$ Safety profiles were generally similar with misoprostol and dinoprostone, except for increased frequency of fetal heart rate abnormalities with misoprostol ( $11 \mathrm{vs} 0 \% ; p=0.03$ ). ${ }^{73}$ It is important to note that many of these trials were not adequately powered for all efficacy and most safety outcomes.

of the six U.S. trials that included misoprostol as a comparator, three assessed vaginal misoprostol in combination with the Foley catheter versus either vaginal or oral misoprostol alone or the Foley catheter alone, and another three trials assessed vaginal misoprostol versus oxytocin or the dinoprostone vaginal insert. The largest U.S. trials identified in the search ( $n=1,308 ; n=1,358$ ) were multicenter trials comparing vaginal misoprostol with vaginal dinoprostone. ${ }^{54,84}$ Compared with the vaginal dinoprostone insert, the $100 \mu \mathrm{g}$ vaginal misoprostol insert was associated with a similar time to vaginal delivery (27.5 vs. 26.6 hours; $p=0.97$ ), while the $200 \mu \mathrm{g}$ misoprostol vaginal insert significantly reduced time to vaginal delivery (32.8 vs. 21.5 hours; $p<0.001$ ). Cesarean delivery rates were similar with the dinoprostone insert and the $100 \mu \mathrm{g}$ (26.4 vs. $27.8 \%$; $p=0.64)$ and $200 \mu \mathrm{g}$ (27.1 vs. $26.0 \% ; p<0.05)$ misoprostol insert doses. All treatments had a similar safety profile; however, the incidence of uterine tachysystole was higher in women receiving the $200 \mu \mathrm{g}$ misoprostol vaginal insert compared with those receiving the dinoprostone insert (13.3 vs. $4.0 \% ; p<0.001$ ). 
In summary, more international trials evaluated misoprostol compared with those conducted in the U.S. Misoprostol is widely used for labor induction both in the U.S. and globally, and in some countries misoprostol may be preferred over other pharmacological methods because it is less expensive and does not require refrigeration. ${ }^{91-93}$ Misoprostol can also be administered through various routes (oral, sublingual, vaginal), which may provide an advantage in hospitals or clinics with less experienced staff. ${ }^{91}$

\section{Trial Population}

The majority of the RCTs included in our search used vague enrollment criteria and typically included a broad population of women presenting at term or postterm for induction of labor, regardless of indication. Required gestational ages ranged from 24 to 42 weeks. Required Bishop's scores ranged from $<4$ to $<8$; some trials specified a cervical dilation of $<2$ or $<3 \mathrm{~cm}$. Of note, lower Bishop's scores (e.g., $<5$ ) and limited dilation at the start of induction have been associated with increased risk of cesarean section following labor induction. ${ }^{94-96}$

Although trials conducted exclusively in women with PROM and PPROM or previous cesarean sections were excluded from our search, a few of the RCTs with very broad patient inclusion criteria included women with ruptured membranes or prior cesarean delivery. A few trials limited the trial population to those with specific indications for labor induction (e.g., hypertension, diabetes, oligohydramnios, postterm pregnancy); thus the results from these trials may be different compared with those that included a broader population of women requiring labor induction. Most trials recruited both nulliparous and multiparous (low and high parity) women; however, very few were powered to detect differences in outcomes between the two groups.

The heterogeneous patient populations in the labor induction trials make it difficult to compare outcomes between trials. Treatment efficacy and safety can vary across the patient population and many factors (e.g., age, parity, indication, medical history) can differentially affect outcomes. Furthermore, as described previously, patient demographic and cultural preferences vary between trial populations. Labor induction rates can also vary; rates in Africa and Asia, where more than half of the RCTs identified in this search were conducted, are approximately 4 and $12 \%$, respectively, compared with approximately $24 \%$ in the U.S. ${ }^{1,88}$ These differences can be attributed to patient and physician factors, as well as availability of adequate facilities and monitoring and the ability to perform a safe cesarean delivery. ${ }^{88}$ Differences among populations within countries may also influence labor induction outcomes. A post hoc analysis of data from a trial comparing the misoprostol vaginal insert with the dinoprostone vaginal insert showed differences in cesarean delivery rates, indications for cesarean delivery, birth weights, and incidences of postpartum hemorrhage among women who identified themselves as black, white, or Hispanic. ${ }^{97}$ Thus, heterogeneity in induction practices and resources, as well as potential disparities based on race and ethnicity, should be considered when interpreting findings from trials of labor induction.

\section{Outcomes Assessments: Efficacy and Safety}

There was wide variability in the reporting of efficacy outcomes between the trials included in our search ( - Table 3 ). The most common primary outcome reported was time from the start of induction to delivery (vaginal or cesarean; $n=28$ trials). Other frequently reported efficacy outcomes were delivery within 24 hours, cesarean deliveries, time to active labor, mode of delivery, and the need for oxytocin; most trials were not adequately powered to assess significant differences in these outcomes. Many trials considered an induction successful if vaginal delivery occurred within 24 hours, yet this timeline may not be realistic considering that the latent phase of labor is longer in women undergoing induction versus spontaneous labor. ${ }^{98,99}$ ACOG recommends longer duration of the latent phase (up to 24 hours or longer) with oxytocin administration for at least 12 to 18 hours after

Table 3 Common prespecified primary and secondary outcome measures in published labor induction trials (all RCTs) ${ }^{23-85}$

\begin{tabular}{|c|c|}
\hline Primary outcomes & Secondary outcomes \\
\hline $\begin{array}{l}\text { - Time to delivery }(n=21) \\
\text { - Time to vaginal delivery }(n=7) \\
\text { - } \text { Delivery } \leq 24 \text { h }(n=6) \\
\text { - Vaginal delivery } \leq 24 \text { h }(n=6) \\
\text { - Rate of cesarean delivery }(n=6) \\
\text { - Rate of vaginal delivery }(n=4) \\
\text { - Time to active labor }(n=4)\end{array}$ & 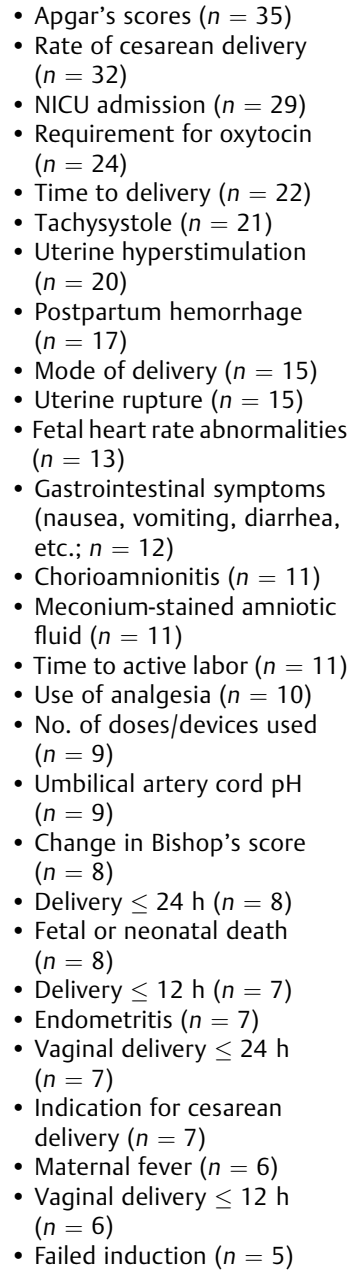 \\
\hline
\end{tabular}

Abbreviations: NICU, neonatal intensive care unit; RCT, randomized controlled trial. 
membrane rupture and a reassuring nonstress test before an induction is considered a failure. ${ }^{99}$ Longer labors can help prevent unnecessary cesarean deliveries which is particularly important in nulliparous women because lowering the rate of primary cesarean delivery rates will in turn reduce repeat cesarean delivery rates. ${ }^{99}$

Nearly all trials included in the search reported at least some safety findings; however, many trials did not include a comprehensive assessment of safety outcomes. In many trials, it was unclear whether any safety outcomes were prespecified. Commonly reported prespecified safety outcomes included maternal and fetal complications (e.g., tachysystole/uterine hyperstimulation, abnormal fetal heart rate tracings, uterine rupture, postpartum hemorrhage) and neonatal outcomes (e.g,. Apgar's scores, arterial cord pH, neonatal intensive care unit [NICU] admission). These safety outcomes were identified as secondary outcomes in the majority of trials and not adequately powered.

\section{Outcomes Assessments: Inconsistent Definitions}

A major limitation of all trials identified in our search was that the definitions of many efficacy and safety parameters (e.g., time to labor, time to delivery, failed induction, uterine hyperstimulation) were inconsistent between trials. For example, most trials defined tachysystole as more than five uterine contractions in a 10-minute period, either with or without fetal heart rate abnormalities; however, other trials defined tachysystole as more than five contractions in 5 minutes, more than 12 contractions in 20 minutes, or failed to define the term altogether. The criteria for failed labor induction also varied between trials, as failed induction was commonly defined as failure to progress to the active phase of labor 12,24 , or 48 hours after treatment initiation; failure to reach the active phase after rupture of membranes and 10 or 12 hours of oxytocin infusion; failure to progress to the active phase despite adequate contractions; or unfavorable cervix following labor induction protocol. Inconsistent definitions for these and other efficacy and safety parameters limit the ability to compare outcomes across trials.

\section{Outcomes Assessments: Clinical Relevancy}

Another major limitation was that in most trials, the primary outcomes were not always the most clinically relevant (e.g., time to delivery, delivery within 24 hours). Duration from induction to delivery might have clinical significance among those with medically indicated delivery before 37 weeks, yet outcomes such as the rate of cesarean delivery or severe maternal or neonatal morbidity are more relevant measures of efficacy and safety. WHO guidelines for labor induction consider cesarean section, severe maternal morbidity or death, perinatal death, and serious neonatal morbidity as top priority outcomes for labor induction trials. ${ }^{4}$ The goal of labor induction should be vaginal delivery because cesarean deliveries are associated with increased risk to the mother and infant, as well as higher health care costs. ${ }^{2,99,100}$ of the 63 RCTs we reviewed, just six used cesarean section rate as a primary outcome measure $40,47,54,64,74,84$; however, two of the trials were not adequately powered.

\section{Outcomes Assessments: Statistical Power}

As noted previously, nearly all RCTs identified in our search were underpowered to detect differences in secondary efficacy and safety outcomes due to small sample sizes. Much larger trial populations are needed to identify statistically significant differences in rare maternal and neonatal adverse events. For example, a clinical trial comparing oral misoprostol with the Foley catheter assessed the noninferiority of misoprostol with respect to the composite outcome of neonatal asphyxia or postpartum hemorrhage. ${ }^{31}$ Assuming that the composite outcome would occur in $13.7 \%$ of patients in the misoprostol group and $12.7 \%$ of patients in the Foley catheter group, it was determined that 1,860 patients (930 women per group) were needed to provide $80 \%$ power to demonstrate noninferiority of misoprostol (prespecified margin of 5\%). A recently completed well-designed clinical trial assessing labor induction versus expectant management in nulliparous women (ARRIVE) enrolled more than 6,000 patients to detect differences in a composite of several severe neonatal morbidity and perinatal mortality outcomes, further highlighting the need for an adequate sample size to ensure sufficient statistical power. ${ }^{18}$ Assuming 92.5\% adherence to the assigned protocol (i.e., labor induction or expectant management) and that the composite outcome of adverse perinatal events would occur in 3.5\% of patients assigned to induction of labor, it was determined that at least 6,000 women were needed to provide $85 \%$ power to detect a $40 \%$ difference in the primary outcome. ${ }^{18}$ While obtaining a sufficiently large sample size is important, it may not always be possible to achieve, given the significant amount of time and resources needed to conduct such a study and this was noted as a limitation by the authors of several published studies.

There are also challenges in defining appropriate assumptions to adequately power trials. In most of the trials included in our search, sample size calculations were based on assumptions derived from previous trials with different trial populations and in some cases, different comparators. Other trials based their assumptions on the investigators' own experience at their institution. Thus, the criteria for demonstrating superiority or noninferiority were fairly subjective.

\section{Outcomes Assessments: Subgroup Analyses}

In addition, of the five trials identified in our search that included prespecified subgroup analyses, ${ }^{25,36,39,81,84}$ only two were powered to detect differences between groups. While subgroup analyses can be useful for evaluating treatment effects in specific patient populations (e.g., nulliparous vs. multiparous women), they should be identified a priori rather than data driven. Furthermore, each subgroup needs to have a large enough sample size to achieve adequate power, which may not always be practical or feasible.

\section{Outcomes Assessments: Patient Satisfaction}

Last, in addition to efficacy and safety, the ideal method for labor induction should minimize patient pain and discomfort. Patient pain and/or satisfaction with the labor induction procedure was assessed in just nine trials (none were 
conducted in the U.S.). ${ }^{30,33,34,42,43,52,53,63,74}$ None of the trials used a validated patient-reported outcome instrument for labor induction; however, until very recently, there have been no validated questionnaires for labor induction. ${ }^{101}$ While WHO and NICE guidelines recommend that patient needs and preferences be taken into consideration when selecting a method of labor induction, ACOG guidelines do not specify that maternal preference be considered. ${ }^{2,4}$ In cases, in which labor induction methods have similar efficacy and safety profiles, we recommend that physicians and health care providers take into account patient preference and overall labor experience. ${ }^{101,102}$

\section{Conclusions and Recommendations for Future Clinical Trial Designs}

The optimal methods for labor induction remain unclear due to the significant limitations in the published literature on labor induction. For many trials, the reported conclusions were not supported by the results. Wide variation in trial design, poorly defined inclusion/exclusion criteria, inconsistent definitions and reporting of outcomes, lack of clinically relevant outcomes, and inadequate statistical power limit the ability for evidencedriven treatment decisions. The majority of trials focused on reducing the duration of labor induction and included primary outcomes related to time to delivery, delivery within 12 to 24 hours, or time to active labor. Clinically important outcomes, including the rate of cesarean delivery and nearly all potential maternal and fetal complications (e.g., uterine hyperstimulation, uterine rupture, abnormal fetal heart rate, postpartum hemorrhage) and neonatal outcomes (e.g., Apgar's scores, NICU admission) were consistently underpowered. In addition, because most of the trials were international, their findings may not be generalizable to many U.S. populations that may differ in terms of age, body mass index (BMI), race/ ethnicity, preference for certain labor induction methods, and labor and delivery practices. Regulatory requirements for the design, conduct, and oversight of clinical trials also varied between countries; it may be difficult to ascertain the validity of results for studies conducted without a governing body to provide standardization and audit. It is important to note that, although the conclusions of this narrative review were based on evidence, as well as our own perspectives and experiences in clinical practice, a systematic review would provide a more rigorous, quantitative assessment of the literature with minimal bias and a comprehensive evaluation of study quality.

Ideally, we recommend that future trials of labor induction methods should be head-to-head RCTs with populations large enough to adequately power primary and secondary outcomes, as well as prespecified subgroup analyses (e.g., parity, BMI, age). Ideally, trials should compare agents with similar mechanisms of action, and combination methods should be compared with the same single agents administered using the same route and dosage. Trials should be multicenter trials with clear unambiguous inclusion/exclusion criteria and consistent protocols for induction. If feasible, trials should be double-blinded but this may not be possible depending on the methods of labor induction used.
All physicians and nurses should follow the guidelines established by ACOG and the Society for Maternal-Fetal Medicine for the safe prevention of primary cesarean delivery. $^{99}$

Future labor induction trials should clearly define efficacy and safety outcomes and use consistent definitions between trials. Trials should report on clinically relevant outcomes because the downstream effects of induction (i.e., cesarean section, maternal and neonatal complications) are more important in determining efficacy and safety. The primary outcome of all labor induction trials should be the rate of cesarean delivery. Cesarean deliveries are associated with increased risk of adverse maternal and neonatal outcomes and are a significant burden to health care systems. ${ }^{99,100}$ The most effective labor induction methods are those that allow safe vaginal delivery, minimizing risk to the mother and infant.

Addressing all these limitations, future clinical trial designs for labor induction will be challenging and may not be possible in some cases. Detecting significant differences in rare adverse events can require thousands of patients and may not be achievable. The management of active labor and delivery based on standard institutional practices will invariably affect all labor induction studies as they are most likely to be pragmatic in nature. Another unavoidable limitation is that many nonclinical factors (e.g., patient preference, obstetrician experience) cannot be regulated through protocol and likely impact labor induction outcomes. Although certain limitations of published labor induction trials may be inevitable, identifying these limitations will hopefully lead to improved trial designs with outcomes that can be better applied to real-world obstetric practice. Given that the best method of labor induction is still being debated and may vary based on indication and other patient characteristics; we recommend that health care providers need to be trained in all labor induction methods to allow for a more personalized approach to labor induction that considers the unique needs and preferences of the patient.

\section{Conflict of Interest}

The authors have nothing to disclose.

\section{Acknowledgments}

Medical writing and editorial assistance for the development of this manuscript were provided by Kimberly Fuller, PhD, of SciFluent Communications, and were financially supported by Ferring Pharmaceuticals, Inc.

\section{References}

1 Martin JA, Hamilton BE, Osterman MJK, Driscoll AK, Drake P. Births: Final Data for 2016. Natl Vital Stat Rep 2018;67(01):1-55

2 ACOG Committee on Practice Bulletins - Obstetrics. ACOG Practice Bulletin No. 107: Induction of labor. Obstet Gynecol 2009;114(2, Pt 1):386-397

3 Rampersad R, Macones GA. Prolonged and postterm pregnancy. In: Gabbe SG, Jauniaux ERM, Niebyl JR, et al, eds. Obstetrics: Normal and Problem Pregnancies. 7th ed. Philadelphia, PA: Elsevier, Inc.; 2017:796-802

4 World Health Organization. WHO recommendations for induction of labour. Geneva, Switzerland: WHO Press; 2011 
5 Dublin S, Johnson KE, Walker RL, et al. Trends in elective labor induction for six United States health plans, 2001-2007.J Womens Health (Larchmt) 2014;23(11):904-911

6 Getahun D. Epidemiologic considerations: scope of problem and disparity concerns. Clin Obstet Gynecol 2014;57(02):326-330

7 Jensen JR, White WM, Coddington CC. Maternal and neonatal complications of elective early-term deliveries. Mayo Clin Proc 2013;88(11):1312-1317

8 Wing DA, Sheibani L. Pharmacotherapy options for labor induction. Expert Opin Pharmacother 2015;16(11):1657-1668

9 Sciscione AC. Methods of cervical ripening and labor induction: mechanical. Clin Obstet Gynecol 2014;57(02):369-376

10 Penfield CA, Wing DA. Labor induction techniques: which is the best? Obstet Gynecol Clin North Am 2017;44(04):567-582

11 Alfirevic Z, Kelly AJ, Dowswell T. Intravenous oxytocin alone for cervical ripening and induction of labour. Cochrane Database Syst Rev 2009;(04):CD003246

12 Cervidil (dinoprostone vaginal insert) [package insert]. Parsippany, NJ: Ferring Pharmaceuticals Inc.; 2016

13 Prepidil (dinoprostone cervical gel) [package insert]. New York, NY: Pharmacia and Upjohn Company; 2016

14 Cytotec (misoprostol oral tablets) [package insert]. New York, NY: G.D. Searle LLC; 2016

15 Wood S, Cooper S, Ross S. Does induction of labour increase the risk of caesarean section? A systematic review and meta-analysis of trials in women with intact membranes. BJOG 2014;121(06): 674-685, discussion 685

16 Mishanina E, Rogozinska E, Thatthi T, Uddin-Khan R, Khan KS, Meads C. Use of labour induction and risk of cesarean delivery: a systematic review and meta-analysis. CMAJ 2014;186(09): 665-673

17 Saccone G, Berghella V. Induction of labor at full term in uncomplicated singleton gestations: a systematic review and metaanalysis of randomized controlled trials. Am J Obstet Gynecol 2015;213(05):629-636

18 Grobman WA, Rice MM, Reddy UM, et al; Eunice Kennedy Shriver National Institute of Child Health and Human Development Maternal-Fetal Medicine Units Network. Labor induction versus expectant management in low-risk nulliparous women. N Engl J Med 2018;379(06):513-523

19 West HM, Jozwiak M, Dodd JM. Methods of term labour induction for women with a previous caesarean section. Cochrane Database Syst Rev 2017;6:CD009792

20 Chen W, Xue J, Peprah MK, et al. A systematic review and network meta-analysis comparing the use of Foley catheters, misoprostol, and dinoprostone for cervical ripening in the induction of labour. BJOG 2016;123(03):346-354

21 Salim R, Schwartz N, Zafran N, Zuarez-Easton S, Garmi G, Romano S. Comparison of single- and double-balloon catheters for labor induction: a systematic review and meta-analysis of randomized controlled trials. J Perinatol 2018;38(03):217-225

22 Knight HE, Cromwell DA, Gurol-Urganci I, Harron K, van der Meulen JH, Smith GCS. Perinatal mortality associated with induction of labour versus expectant management in nulliparous women aged 35 years or over: an English national cohort study. PLoS Med 2017;14(11):e1002425

23 Conde A, Ben S, Tarigo J, et al. Comparison between vaginal and sublingual misoprostol $50 \mu \mathrm{g}$ for cervical ripening prior to induction of labor: randomized clinical trial. Arch Gynecol Obstet 2017;295(04):839-844

24 Rouzi AA, Alsahly N, Alamoudi R, et al. Randomized clinical trial between hourly titrated and 2 hourly static oral misoprostol solution for induction of labor. Am J Obstet Gynecol 2017;216 (04):405.e1-405.e6

25 Schoen CN, Grant G, Berghella V, Hoffman MK, Sciscione A. Intracervical Foley catheter with and without oxytocin for labor induction: a randomized controlled trial. Obstet Gynecol 2017; 129(06):1046-1053
26 Aduloju OP, Akintayo AA, Adanikin AI, Ade-Ojo IP. Combined Foley's catheter with vaginal misoprostol for pre-induction cervical ripening: a randomised controlled trial. Aust $\mathrm{N} \mathrm{Z} \mathrm{J}$ Obstet Gynaecol 2016;56(06):578-584

27 Connolly KA, Kohari KS, Rekawek P, et al. A randomized trial of Foley balloon induction of labor trial in nulliparas (FIAT-N). Am J Obstet Gynecol 2016;215(03):392.e1-392.e6

28 Garba I, Muhammed AS, Muhammad Z, Galadanci HS, Ayyuba R, Abubakar IS. Induction to delivery interval using transcervical Foley catheter plus oxytocin and vaginal misoprostol: a comparative study at Aminu Kano Teaching Hospital, Kano, Nigeria. Ann Afr Med 2016;15(03):114-119

29 Levine LD, Downes KL, Elovitz MA, Parry S, Sammel MD, Srinivas SK. Mechanical and pharmacologic methods of labor induction: a randomized controlled trial. Obstet Gynecol 2016;128(06): $1357-1364$

30 Sayed Ahmed WA, Ibrahim ZM, Ashor OE, Mohamed ML, Ahmed MR, Elshahat AM. Use of the Foley catheter versus a double balloon cervical ripening catheter in pre-induction cervical ripening in postdate primigravidae. J Obstet Gynaecol Res 2016;42(11):1489-1494

31 Ten Eikelder ML, Oude Rengerink K, Jozwiak M, et al. Induction of labour at term with oral misoprostol versus a Foley catheter (PROBAAT-II): a multicentre randomised controlled non-inferiority trial. Lancet 2016;387(10028):1619-1628

32 Yenuberi H, Abraham A, Sebastian A, Benjamin SJ, Jeyaseelan V, Mathews JE. A randomised double-blind placebo-controlled trial comparing stepwise oral misoprostol with vaginal misoprostol for induction of labour. Trop Doct 2016;46(04):198-205

33 Chavakula PR, Benjamin SJ, Abraham A, Londhe V, Jeyaseelan V, Mathews JE. Misoprostol versus Foley catheter insertion for induction of labor in pregnancies affected by fetal growth restriction. Int J Gynaecol Obstet 2015;129(02):152-155

34 Ezechukwu PC, Ugwu EO, Obi SN, Chigbu CO. Oral versus vaginal misoprostol for induction of labor in Enugu, Nigeria: a randomized controlled trial. Arch Gynecol Obstet 2015;291(03):537-544

35 Kehl S, Ziegler J, Schleussner E, et al. Sequential use of doubleballoon catheter and oral misoprostol versus oral misoprostol alone for induction of labour at term (CRBplus trial): a multicentre, open-label randomised controlled trial. BJOG 2015;122 (01):129-136

36 Løkkegaard E, Lundstrøm M, Kjær MM, Christensen IJ, Pedersen HB, Nyholm H. Prospective multi-centre randomised trial comparing induction of labour with a double-balloon catheter versus dinoprostone. J Obstet Gynaecol 2015;35(08):797-802

37 Shechter-Maor G, Haran G, Sadeh-Mestechkin D, Ganor-Paz Y, Fejgin MD, Biron-Shental T. Intra-vaginal prostaglandin E2 versus double-balloon catheter for labor induction in term oligohydramnios. J Perinatol 2015;35(02):95-98

38 Sheela CN, John C, Preethi R. Comparison of the efficacy and safety of sublingual misoprostol with that of vaginal misoprostol for labour induction at term. J Obstet Gynaecol 2015;35(05):469-471

39 Edwards RK, Szychowski JM, Berger JL, et al. Foley catheter compared with the controlled-release dinoprostone insert: a randomized controlled trial. Obstet Gynecol 2014;123(06): $1280-1287$

40 Jozwiak M, ten Eikelder M, Oude Rengerink K, et al; PROBAAT Study Group. Foley catheter versus vaginal misoprostol: randomized controlled trial (PROBAAT-M study) and systematic review and meta-analysis of literature. Am J Perinatol 2014;31(02):145-156

41 Lanka S, Surapaneni T, Nirmalan PK. Concurrent use of Foley catheter and misoprostol for induction of labor: a randomized clinical trial of efficacy and safety. J Obstet Gynaecol Res 2014;40 (06):1527-1533

42 Mei-Dan E, Walfisch A, Valencia C, Hallak M. Making cervical ripening EASI: a prospective controlled comparison of single versus double balloon catheters. J Matern Fetal Neonatal Med 2014;27(17):1765-1770 
43 Reinhard J, Rösler R, Yuan J, et al. Prostaglandin E2 labour induction with intravaginal (Minprostin) versus intracervical (Prepidil) administration at term: randomized study of maternal and neonatal outcome and patient's perception using the osgood semantic differential scales. BioMed Res Int 2014;2014:682919

44 Rouzi AA, Alsibiani S, Mansouri N, Alsinani N, Darhouse K. Randomized clinical trial between hourly titrated oral misoprostol and vaginal dinoprostone for induction of labor. Am J Obstet Gynecol 2014;210(01):56.e1-56.e6

45 Sharami SH, Milani F, Faraji R, et al. Comparison of $25 \mu \mathrm{g}$ sublingual and $50 \mu \mathrm{g}$ intravaginal misoprostol for cervical ripening and labor: a randomized controlled equivalence trial. Arch Iran Med 2014;17(10):652-656

46 Suffecool K, Rosenn BM, Kam S, Mushi J, Foroutan J, Herrera K. Labor induction in nulliparous women with an unfavorable cervix: double balloon catheter versus dinoprostone. J Perinat Med 2014;42(02):213-218

47 Wang W, Zheng J, Fu J, et al. Which is the safer method of labor induction for oligohydramnios women? Transcervical double balloon catheter or dinoprostone vaginal insert. J Matern Fetal Neonatal Med 2014;27(17):1805-1808

48 Aalami-Harandi R, Karamali M, Moeini A. Induction of labor with titrated oral misoprostol solution versus oxytocin in term pregnancy: randomized controlled trial. Rev Bras Ginecol Obstet 2013;35(02):60-65

49 Carbone JF, Tuuli MG, Fogertey PJ, Roehl KA, Macones GA. Combination of Foley bulb and vaginal misoprostol compared with vaginal misoprostol alone for cervical ripening and labor induction: a randomized controlled trial. Obstet Gynecol 2013; 121(2, Pt 1):247-252

50 Koc O, Duran B, Ozdemirci S, Albayrak M, Koc U. Oxytocin versus sustained-release dinoprostone vaginal pessary for labor induction of unfavorable cervix with Bishop score $\geq 4$ and $\leq 6$ : a randomized controlled trial. J Obstet Gynaecol Res 2013;39(04): 790-798

51 Rahman H, Pradhan A, Kharka L, Renjhen P, Kar S, Dutta S. Comparative evaluation of 50 microgram oral misoprostol and 25 microgram intravaginal misoprostol for induction of labour at term: a randomized trial. J Obstet Gynaecol Can 2013;35(05): 408-416

52 Souza AS, Feitosa FE, Costa AA, et al. Titrated oral misoprostol solution versus vaginal misoprostol for labor induction. Int $\mathrm{J}$ Gynaecol Obstet 2013;123(03):207-212

53 Ugwu EO, Onah HE, Obi SN, et al. Effect of the Foley catheter and synchronous low dose misoprostol administration on cervical ripening: a randomised controlled trial. J Obstet Gynaecol 2013; 33(06):572-577

54 Wing DA, Brown R, Plante LA, Miller H, Rugarn O, Powers BL. Misoprostol vaginal insert and time to vaginal delivery: a randomized controlled trial. Obstet Gynecol 2013;122(2, Pt 1):201-209

55 Akay NO, Hızlı D, Yılmaz SS, Yalvaç S, Kandemir O. Comparison of low-dose oxytocin and dinoprostone for labor induction in postterm pregnancies: a randomized controlled prospective study. Gynecol Obstet Invest 2012;73(03):242-247

56 Chitrakar NS. Comparison of misoprostol versus dinoprostone for pre-induction cervical ripening at-term. J Nepal Health Res Counc 2012;10(01):10-15

57 Cromi A, Ghezzi F, Uccella S, et al. A randomized trial of preinduction cervical ripening: dinoprostone vaginal insert versus double-balloon catheter. Am J Obstet Gynecol 2012;207 (02):125.e1-125.e7

58 Fitzpatrick CB, Grotegut CA, Bishop TS, Canzoneri BJ, Heine RP, Swamy GK. Cervical ripening with foley balloon plus fixed versus incremental low-dose oxytocin: a randomized controlled trial. J Matern Fetal Neonatal Med 2012;25(07):1006-1010

59 Kandil M, Emarh M, Sayyed T, Masood A. Foley catheter versus intra-vaginal misoprostol for induction of labor in post-term gestations. Arch Gynecol Obstet 2012;286(02):303-307
60 Mei-Dan E, Walfisch A, Suarez-Easton S, Hallak M. Comparison of two mechanical devices for cervical ripening: a prospective quasi-randomized trial. J Matern Fetal Neonatal Med 2012;25 (06):723-727

61 Thaisomboon A, Russameecharoen K, Wanitpongpan P, Phattanachindakun B, Changnoi A. Comparison of the efficacy and safety of titrated oral misoprostol and a conventional oral regimen for cervical ripening and labor induction. Int J Gynaecol Obstet 2012;116(01):13-16

62 Balci O, Mahmoud AS, Acar A, Colakoglu MC. Comparison of induction of labor with vaginal misoprostol plus oxytocin versus oxytocin alone in term primigravidae. J Matern Fetal Neonatal Med 2011;24(09):1084-1087

63 Güngördük K, Yildirim G, Güngördük O, Ark C, Tekirdağ I. Sustained-release dinoprostone vaginal pessary with concurrent high-dose oxytocin infusion compared to sustained-release dinoprostone vaginal pessary followed 6 h later by high-dose oxytocin infusion for labor induction in women at term with unfavorable cervix: a randomized controlled trial. Gynecol Obstet Invest 2011;71(01):32-40

64 Jozwiak M, Oude Rengerink K, Benthem M, et al; PROBAAT Study Group. Foley catheter versus vaginal prostaglandin E2 gel for induction of labour at term (PROBAAT trial): an open-label, randomised controlled trial. Lancet 2011;378(9809):2095-2103

65 Kehl S, Ehard A, Berlit S, Spaich S, Sütterlin M, Siemer J. Combination of misoprostol and mechanical dilation for induction of labour: a randomized controlled trial. Eur J Obstet Gynecol Reprod Biol 2011;159(02):315-319

66 Saeed GA, Fakhar S, Nisar N, Alam AY. Misoprostol for term labor induction: a randomized controlled trial. Taiwan J Obstet Gynecol 2011;50(01):15-19

67 Salim R, Zafran N, Nachum Z, Garmi G, Kraiem N, Shalev E. Singleballoon compared with double-balloon catheters for induction of labor: a randomized controlled trial. Obstet Gynecol 2011;118 (01):79-86

68 Saxena P, Puri M, Bajaj M, Mishra A, Trivedi SS. A randomized clinical trial to compare the efficacy of different doses of intravaginal misoprostol with intracervical dinoprostone for cervical ripening and labor induction. Eur Rev Med Pharmacol Sci 2011; 15(07):759-763

69 Balci O, Mahmoud AS, Ozdemir S, Acar A. Induction of labor with vaginal misoprostol plus oxytocin versus oxytocin alone. Int $\mathrm{J}$ Gynaecol Obstet 2010;110(01):64-67

70 Moraes Filho OB, Albuquerque RM, Cecatti JG. A randomized controlled trial comparing vaginal misoprostol versus Foley catheter plus oxytocin for labor induction. Acta Obstet Gynecol Scand 2010;89(08):1045-1052

71 Tan TC, Yan SY, Chua TM, Biswas A, Chong YS. A randomised controlled trial of low-dose misoprostol and dinoprostone vaginal pessaries for cervical priming. BJOG 2010;117(10): $1270-1277$

72 Hill JB, Thigpen BD, Bofill JA, Magann E, Moore LE, Martin JN Jr. A randomized clinical trial comparing vaginal misoprostol versus cervical Foley plus oral misoprostol for cervical ripening and labor induction. Am J Perinatol 2009;26(01):33-38

73 Ozkan S, Calişkan E, Doğer E, Yücesoy I, Ozeren S, Vural B. Comparative efficacy and safety of vaginal misoprostol versus dinoprostone vaginal insert in labor induction at term: a randomized trial. Arch Gynecol Obstet 2009;280(01):19-24

74 Pennell CE, Henderson JJ, O'Neill MJ, McChlery S, Doherty DA, Dickinson JE. Induction of labour in nulliparous women with an unfavourable cervix: a randomised controlled trial comparing double and single balloon catheters and PGE2 gel. BJOG 2009; 116(11):1443-1452

75 Zahran KM, Shahin AY, Abdellah MS, Elsayh KI. Sublingual versus vaginal misoprostol for induction of labor at term: a randomized prospective placebo-controlled study. J Obstet Gynaecol Res 2009;35(06):1054-1060 
76 Abbassi RM, Sirichand P, Rizvi S. Safety and efficacy of oral versus vaginal misoprostol use for induction of labour at term. J Coll Physicians Surg Pak 2008;18(10):625-629

77 Calder AA, Loughney AD, Weir CJ, Barber JW. Induction of labour in nulliparous and multiparous women: a UK, multicentre, open-label study of intravaginal misoprostol in comparison with dinoprostone. BJOG 2008;115(10):1279-1288

78 Cheng SY, Ming H, Lee JC. Titrated oral compared with vaginal misoprostol for labor induction: a randomized controlled trial. Obstet Gynecol 2008;111(01):119-125

79 Fonseca L, Wood HC, Lucas MJ, et al. Randomized trial of preinduction cervical ripening: misoprostol vs oxytocin. Am J Obstet Gynecol 2008;199(03):305.e1-305.e5

80 Krithika KS, Aggarwal N, Suri V. Prospective randomised controlled trial to compare safety and efficacy of intravaginal misoprostol with intracervical cerviprime for induction of labour with unfavourable cervix. J Obstet Gynaecol 2008;28 (03):294-297

81 Pettker CM, Pocock SB, Smok DP, Lee SM, Devine PC. Transcervical Foley catheter with and without oxytocin for cervical ripening: a randomized controlled trial. Obstet Gynecol 2008;111 (06):1320-1326

82 Prager M, Eneroth-Grimfors E, Edlund M, Marions L. A randomised controlled trial of intravaginal dinoprostone, intravaginal misoprostol and transcervical balloon catheter for labour induction. BJOG 2008;115(11):1443-1450

83 Tanir HM, Sener T, Yildiz C, Kaya M, Kurt I. A prospective randomized trial of labor induction with vaginal controlledrelease dinoprostone inserts with or without oxytocin and misoprostol+oxytocin. Clin Exp Obstet Gynecol 2008;35(01): 65-68

84 Wing DA; Misoprostol Vaginal Insert Consortium. Misoprostol vaginal insert compared with dinoprostone vaginal insert: a randomized controlled trial. Obstet Gynecol 2008;112(04): 801-812

85 Zvandasara P, Saungweme G, Mlambo J, Chidembo W, Madzivanzira N, Mwanjira C. Induction of labour with titrated oral misoprostol suspension. A comparative study with vaginal misoprostol. Cent Afr J Med 2008;54(9-12):43-49

86 Lajusticia H, Martínez-Domínguez SJ, Pérez-Roncero GR, Chedraui P, Pérez-López FR; Health Outcomes and Systematic Analyses (HOUSSAY) Project. Single versus double-balloon catheters for the induction of labor of singleton pregnancies: a meta-analysis of randomized and quasi-randomized controlled trials. Arch Gynecol Obstet 2018;297(05):1089-1100

87 National Institute for Health and Care Excellence. Inducing labor (NICE guideline CG70). 2008. Available from: https://www.nice. org.uk/Guidance/CG70. Accessed October 30, 2018

88 Vogel JP, Souza JP, Gülmezoglu AM. Patterns and outcomes of induction of labour in Africa and Asia: a secondary analysis of the WHO global survey on maternal and neonatal health. PLoS One 2013;8(06):e65612

89 Vogel JP, Gülmezoglu AM, Hofmeyr GJ, Temmerman M. Global perspectives on elective induction of labor. Clin Obstet Gynecol 2014;57(02):331-342

90 Guerra GV, Cecatti JG, Souza JP, et al; WHO Global Survey on Maternal Perinatal Health in Latin America Study Group. Elective induction versus spontaneous labour in Latin America. Bull World Health Organ 2011;89(09):657-665

91 Lang DL, Zhao FL, Robertson J. Prevention of postpartum haemorrhage: cost consequences analysis of misoprostol in lowresource settings. BMC Pregnancy Childbirth 2015;15:305

92 Nadia Bennett K, Park H, Cioffi J, Calixte R, Vintzileos A. A comparison of obstetrical outcomes and costs between misoprostol and dinoprostone for induction of labor. J Matern Fetal Neonatal Med 2016;29(22):3732-3736

93 Bierut A, Dowgiałło-Smolarczyk J, Pieniążek I, et al. Misoprostol vaginal insert in labor induction: a cost-consequences model for 5 European countries-an economic evaluation supported with literature review and retrospective data collection. Adv Ther 2016;33(10):1755-1770

94 Vrouenraets FP, Roumen FJ, Dehing CJ, van den Akker ES, Aarts MJ, Scheve EJ. Bishop score and risk of cesarean delivery after induction of labor in nulliparous women. Obstet Gynecol 2005; 105(04):690-697

95 Verhoeven CJ, Oudenaarden A, Hermus MA, Porath MM, Oei SG, Mol BW. Validation of models that predict cesarean section after induction of labor. Ultrasound Obstet Gynecol 2009;34(03): 316-321

96 Verhoeven CJ, van Uytrecht CT, Porath MM, Mol BW. Risk factors for cesarean delivery following labor induction in multiparous women. J Pregnancy 2013;2013:820892

97 Stephenson ML, Hawkins JS, Pevzner L, Powers BL, Wing DA. Racial/ ethnic differences in labor outcomes with prostaglandin vaginal inserts. J Racial Ethn Health Disparities 2015;2(02):149-157

98 Schoen C, Navathe R. Failed induction of labor. Semin Perinatol 2015;39(06):483-487

99 American College of Obstetricians and Gynecologists; Society for Maternal-Fetal Medicine. Obstetric care consensus no. 1: safe prevention of the primary cesarean delivery. Obstet Gynecol 2014;123(03):693-711

100 Truven Health Analytics. The cost of having a baby in the United States. 2013

101 Ten Eikelder ML, van de Meent MM, Mast K, et al. Women's experiences with and preference for induction of labor with oral misoprostol or Foley catheter at term. Am J Perinatol 2017;34 (02):138-146

102 Schwarz C, Gross MM, Heusser P, Berger B. Women's perceptions of induction of labour outcomes: results of an online-survey in Germany. Midwifery 2016;35:3-10 